\title{
An XMM-Newton view of the X-ray flat radio-quiet quasar PG 1416-129
}

\author{
D. Porquet ${ }^{1}$, J. N. Reeves ${ }^{2,3}$, A. Markowitz ${ }^{2, \star}$, T. J. Turner ${ }^{2,5}$, L. Miller ${ }^{6}$, and K. Nandra ${ }^{7}$ \\ 1 Max-Plank-Institut für Extraterrestrische Physik, Postfach 1312, 85741 Garching, Germany \\ e-mail: dporquet@mpe.mpg.de \\ 2 Laboratory for High Energy Astrophysics, Code 662, NASA Goddard Space Flight Center, Greenbelt, MD 20771, USA \\ 3 Dept. of Physics and Astronomy, Johns Hopkins University, 3400 N. Charles Street, Baltimore, MD 21218, USA \\ 4 Dept. of Physics, University of Maryland Baltimore County, 1000 Hilltop Circle, Baltimore, MD 21250, USA \\ 5 Dept. of Physics, University of Oxford, Denys Wilkinson Building, Keble Road, Oxford OX1 3RH, UK \\ ${ }^{6}$ Astrophysics Group, Imperial College London, Blackett Laboratory, Prince Consort Road, London SW7 2AW, UK
}

Received 15 July 2006 / Accepted 20 November 2006

\section{ABSTRACT}

\begin{abstract}
Aims. The radio-quiet quasar PG 1416-129 $(z=0.129)$ exhibits atypical optical and X-ray properties. Between 1990 and 2000 , in response to its optical continuum decrease, the "classical" broad component of $\mathrm{H} \beta$ almost completely disappeared, with a factor of 10 decrease in the line flux. In addition, the width of the broad component of the $\mathrm{H} \beta$ line decreased significantly from $4000 \mathrm{~km} \mathrm{~s}{ }^{-1}$ to $1450 \mathrm{~km} \mathrm{~s}^{-1}$. In the X-ray band, this object was observed by Ginga in 1988 to have the hardest quasar photon index, with $\Gamma=1.1 \pm 0.1$. We present an XMM-Newton/EPIC observation of PG 1416-129 performed in July 2004.

Methods. We analyze the time-averaged pn spectrum of this quasar, as well as perform time-resolved spectroscopy.

Results. We find that during the present XMM-Newton observation, PG 1416-129 still has a rather hard photon index, both in the soft $(0.2-2 \mathrm{keV})$ and hard $(2-12 \mathrm{keV})$ energy ranges, compared to radio-quiet quasars (BLS1 and NLS1) but compatible with the photon index value found for radio-loud quasars. This object also shows long-term luminosity variability over 16 years by a factor of three with a variation of photon index from $\sim 1.2$ to $\sim 1.8$. In the soft energy band $(0.2-2 \mathrm{keV})$, we found a very weak soft X-ray excess compared to other RQ quasars. The whole time averaged spectrum is fit very well either by X-ray ionized reflection from the accretion disk surface, by a warm absorber-emitter plus power-law, or by a smeared absorption/emission from a relativistic outflow. While no constant narrow Fe $\mathrm{K}$ line at $6.4 \mathrm{keV}$ is observed, we find the possible presence of two non-simultaneous transient iron lines: a redshifted narrow iron line at about $5.5 \mathrm{keV}$ (96.4\% confidence level according to multi-trial Monte-Carlo simulations) at the beginning of this observation and the appearance of a line at $6.3-6.4 \mathrm{keV}(99.1 \%$ c.l.) at the end of the observation. These variable lines could be generated by discrete hot-spots on the accretion disk surface.
\end{abstract}

Key words. galaxies: active - X-rays: galaxies - accretion, accretion discs - quasars: individual: PG 1416-129

\section{Introduction}

PG 1416-129 $(z=0.129)$ was one of the three brightest lowredshift $(z<0.2)$ radio-quiet quasars observed during the ASCA mission (Reeves \& Turner 2000). It has a high bolometric luminosity, $10^{46} \mathrm{erg} \mathrm{s}^{-1}$ (Vestergaard 2002), and a black hole mass of about 3-6 × $10^{8} M_{\odot}$ (Vestergaard 2002; Hao et al. 2005). This object has an accretion rate with respect to Eddington of about 0.12 (Hao et al. 2005). It has been associated with the 2MASS object at $\alpha_{J 2000}=14^{\mathrm{h}} 19^{\mathrm{m}} 03^{\mathrm{s}} .81, \delta_{J 2000}=-13^{\circ} 10^{\prime} 44^{\prime \prime} \cdot 7$ (Barkhouse \& Hall 2001). It has strong, broad permitted optical emission lines: $F W H M(\mathrm{H} \beta)=6110 \mathrm{~km} \mathrm{~s}^{-1}$ (Boroson \& Green 1992), but the measurement of this width was contaminated by the presence of a very broad component, as shown by Sulentic et al. (2000), who measured $F W H M(\mathrm{H} \beta)=4000 \mathrm{~km} \mathrm{~s}^{-1}$. This value of the line width led to it being described as a BroadLine $(\mathrm{BL})$ AGN $\left(F W H M(\mathrm{H} \beta)>2000 \mathrm{~km} \mathrm{~s}^{-1}\right)$. However, observations of this broad-line quasar have revealed a significant drop-off in its continuum luminosity by about a factor of four at $4500 \AA$ between 1990 and 2000 (Sulentic et al. 2000). In response to this continuum decrease, the "classical" broad component of $\mathrm{H} \beta$ has almost completely disappeared, with a factor

\footnotetext{
^ NASA Postdoc Program Associate.
}

of 10 decrease in the line flux (Sulentic et al. 2000). Additionally, the width of the broad component of the $\mathrm{H} \beta$ line decreased significantly over those 10 years, from $4000 \mathrm{~km} \mathrm{~s}^{-1}$ to $1450 \mathrm{~km} \mathrm{~s}^{-1}$.

In the X-ray band, PG 1416-129 shows peculiar behavior compared to other low-redshift quasars. A spectrum obtained by Ginga in 1988 showed the hardest spectral index $(\Gamma=1.1 \pm 0.1)$ of all known quasars in the $2-20 \mathrm{keV}$ energy range (Williams et al. 1992). The Compton Gamma-ray Observatory/OSSE observations in 1994 showed that PG 1416-129 has a very steep spectrum $(\Gamma=3.2 \pm 0.5)$ above $50 \mathrm{keV}$, and consequently the most dramatic cut-off between $50 \mathrm{keV}$ and $100 \mathrm{keV}$ (Staubert \& Maisack 1996). However, from an ASCA observation in 1994, Reeves \& Turner (2000) found a photon index for this object of $\Gamma=1.78 \pm 0.02$, which is closer to the values found in most radio-quiet low-redshift quasars (e.g., Porquet et al. 2004a) than the very hard photon index measured previously with Ginga. In addition, during this ASCA observation, a possible $\mathrm{Fe} \mathrm{K} \alpha$ line was detected at $E=6.54_{-0.18}^{+0.16} \mathrm{keV}(E W=140 \pm 75 \mathrm{eV})$ with an F-test probability of $98.3 \%$. This object also shows long-term flux variability as reported in Table 1 . In this paper we report the analysis of the XMM-Newton observation of PG 1416-129 performed in July 2004. In addition to the previous X-ray observations already published and the present XMM-Newton 
Table 1. Summary of the power-law index and luminosities $\left(\times 10^{44} \mathrm{erg} \mathrm{s}^{-1}\right)$ observed by different X-ray satellites in the $2-10 \mathrm{keV}$ energy range over 16 years (5 observations). For a direct comparison, the luminosities have been re-calculated when needed assuming $H_{0}=75 \mathrm{~km} \mathrm{~s}^{-1} \mathrm{Mpc}^{-1}$ and $q_{0}=0.5$. The references (Col. 5) correspond to: RT00 (Reeves \& Turner 2000), LT97 (Lawson \& Turner 1997).

\begin{tabular}{llcll}
\hline \hline Satellites & $\begin{array}{l}\text { Obs. date } \\
\text { (dd/mm/yyyy })\end{array}$ & $\Gamma$ & Lum & Ref. \\
\hline Ginga & $02 / 02 / 1988$ & $1.18 \pm 0.06$ & 1.4 & LT97 \\
Ginga & $21 / 09 / 1991$ & $1.48 \pm 0.13$ & 1.2 & LT97 \\
ASCA & $29 / 07 / 1994$ & $1.78 \pm 0.02$ & 3.0 & RT00 \\
RXTE & $21 / 08 / 1998$ & $1.17 \pm 0.15$ & 1.0 & This work \\
XMM-Newton & $14 / 07 / 2004$ & $1.56 \pm 0.04$ & 0.98 & This work \\
\hline
\end{tabular}

observation, we also show the currently unpublished 1998 RXTE data. Details of the RXTE data analysis are reported in Appendix A.

\section{XMM-Newton observation and data reduction}

XMM-Newton observed PG 1416-129 on July 14, 2004 (ID 0203770201; orbit 842; exposure time $\sim 50 \mathrm{ks}$ ). The EPIC-MOS cameras (Turner et al. 2001) operated in the Small Window mode, while the EPIC-pn camera (Strüder et al. 2001) was operated in the standard Full Frame mode. The data were reprocessed and cleaned (net pn time exposure $\sim 45 \mathrm{ks}$ ) using the XMM-Newton SAS VERSION 6.5.0 (Science Analysis Software) package. Since the effect of pile-up was negligible, X-ray events corresponding to patterns $0-12$ and $0-4$ events (single and double pixels) were selected for the MOS and pn, respectively. Only good X-ray events (with FLAG $=0$ ) were included. The lowenergy cutoff was set to 300 and $200 \mathrm{eV}$ for MOS and pn, respectively. In this article we present the pn results only, since the pn-CCD have a better sensitivity over a broader energy range $(0.2-12 \mathrm{keV})$ compared to the MOS CCDs. However, we verified that similar results were obtained using the MOS data. The pn source spectra were extracted using a circular region of diameter $30^{\prime \prime}$ centered on the source position. PG 1416-129 is by far the brightest X-ray source in this $30^{\prime}$ EPIC field-of-view. Background spectra were taken from a box region $\left(7.3^{\prime} \times 4^{\prime}\right)$ on the same CCD as the source (excluding X-ray point sources). The XSPEC V11.3 software package (Arnaud 1996) was used for spectral analysis of the background-subtracted spectrum, using the response matrices and ancillary files derived from the SAS tasks RMFGEN and ARFGEN. The pn spectrum was binned to give a minimum of 50 counts per bin.

The signal to noise ratio was not sufficient for reliable RGS data analysis, and the OM was blocked during this observation.

\section{XMM-Newton spectral analysis}

In all fits, we included both the Galactic column density $\left(N_{\mathrm{H}}^{\mathrm{Gal}}=\right.$ $6.80 \times 10^{20} \mathrm{~cm}^{-2}$, obtained from the COLDENS program using the compilations of Dickey \& Lockman 1990), and a possible additional absorption component located at the quasar redshift $\left(N_{\mathrm{H}}^{\mathrm{int}}\right)$. Note that all fit parameters are given in the quasar's rest frame, with values of $H_{0}=75 \mathrm{~km} \mathrm{~s}^{-1} \mathrm{Mpc}^{-1}$ and $q_{0}=0.5$ assumed throughout. The errors quoted correspond to $90 \%$ confidence ranges for one interesting parameter $\left(\Delta \chi^{2}=2.71\right)$. Abundances are those of Anders \& Grevesse (1989). In the following, we use the updated cross-sections for X-ray absorption by the interstellar medium (TBABS in XSPEC) from Wilms et al. (2000).

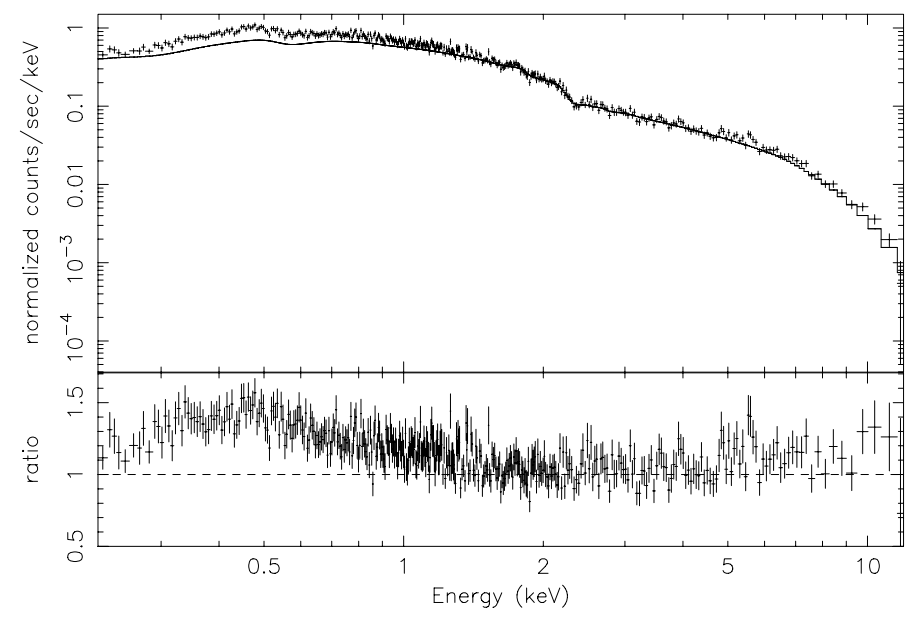

Fig. 1. The pn spectrum of PG 1416-129 (in the observed frame). A power-law has been fit to the $2-5 \mathrm{keV}$ data and extrapolated to lower and higher energies. A weak soft X-ray positive residual is seen between $0.3-1 \mathrm{keV}$, as well as the presence of a $\mathrm{Fe} \mathrm{K} \alpha$ complex between about $4.8-5.7 \mathrm{keV}(\sim 5.4-6.4 \mathrm{keV}$ in the quasar frame). For presentation only, the data have been re-binned into groups of 10 bins, after a grouping of a minimum of 20 counts per bin is used for the fit.

\subsection{The time averaged spectrum}

We found an average pn count rate in the $0.2-12 \mathrm{keV}$ energy range of $1.36 \pm 0.01 \mathrm{cts} \mathrm{s}^{-1}$. First, fitting the overall $0.2-12 \mathrm{keV}$ pn spectrum with a single absorbed power-law model, a moderately acceptable fit was found ( $\chi_{\text {red }}^{2}=1.18$ for 979 d.o.f.), with $\Gamma=1.75 \pm 0.01$. To characterize the hard X-ray continuum, we fit an absorbed power-law model over the $2-5 \mathrm{keV}$ energy range, where the spectrum should be relatively unaffected by the presence of a broad soft excess, a Warm Absorber-Emitter medium, an $\mathrm{Fe} \mathrm{K} \alpha$ emission line, and a contribution above $8 \mathrm{keV}$ from a high energy Compton reflection hump. In this energy range, the data are well fit by a single power-law model with $\Gamma=1.61 \pm 0.06$ $\left(\chi^{2} /\right.$ d.o.f. $\left.=346.2 / 377\right)$. This power-law index is similar to values found over the same energy range for radio-loud quasars $(\langle\Gamma=1.74\rangle$ with a standard deviation of 0.03 ; Porquet et al. $2004 a)$, but harder than the values commonly seen in radio-quiet quasars: $\langle\Gamma=1.90\rangle$ (with a standard deviation of 0.27 ) and $\langle\Gamma=2.37\rangle$ (with a standard deviation of 0.11 ) for Broad Line quasars and Narrow Line quasars, respectively (Porquet et al. 2004a). A similar conclusion is found when comparing with the average power law index measured in radio-quiet quasars above $2 \mathrm{keV}\left(\left\langle\Gamma_{2-10 \mathrm{keV}}\right\rangle=2\right.$ with a dispersion of 0.25 , George et al. 2000; $\left\langle\Gamma_{2-12 \mathrm{keV}}\right\rangle=1.89 \pm 0.11$, Piconcelli et al. 2005).

Figure 1 displays the spectrum when the power-law model is extrapolated over the $0.2-12 \mathrm{keV}$ broad band energy range. A weak positive residual is seen below $1 \mathrm{keV}$ due to the presence of a soft X-ray excess. In addition, a Fe K $\alpha$ complex between about $4.8-5.7 \mathrm{keV}(\sim 5.4-6.4 \mathrm{keV}$ in the quasar frame) seems to be present.

\subsubsection{The soft spectrum: $0.2-2 \mathrm{keV}$}

First we fit the $0.2-2 \mathrm{keV}$ energy range with a single absorbed power-law, and found a good fit with $N_{\mathrm{H}}^{\text {int }}=2.1 \pm 0.7 \times 10^{20} \mathrm{~cm}^{-2}$ and a photon index of $\Gamma=1.96 \pm 0.05\left(\chi^{2} /\right.$ d.o.f. $\left.=364.9 / 355\right)$. This value is compatible within the error bars with $\Gamma=2.2 \pm 0.2$ inferred from a ROSAT PSPC observation performed in 1992 (de Kool \& Meurs 1994). This value is marginally smaller than 
Table 2. Best-fit spectral parameters of the time-averaged pn spectrum in the $2-12 \mathrm{keV}$ energy range with an absorbed (Galactic, $N_{\mathrm{H}}=6.8 \times$ $10^{20} \mathrm{~cm}^{-2}$ ) power-law (PL) component plus a line profile: zgauss: Gaussian profile; and DISKLINE and LAOR: line profile emitted by a relativistic accretion disk for a non-rotating black hole (Fabian et al. 1989) and a maximally rotating black hole (Laor 1991), respectively. The line fluxes are expressed in $10^{-6}$ photons $\mathrm{cm}^{-2} \mathrm{~s}^{-1}$. We assume an emissivity index $q$ equal to -2 . (a): $R_{\mathrm{in}}=6 R_{\mathrm{g}}$ and $R_{\text {out }}=1000 R_{\mathrm{g}}$. (b): $R_{\mathrm{in}}=1.235 R_{\mathrm{g}}$ and $R_{\text {out }}=400 R_{\mathrm{g}}$.

\begin{tabular}{|c|c|c|c|c|c|c|c|c|}
\hline \multirow[t]{2}{*}{ Model } & \multirow[t]{2}{*}{$\Gamma$} & \multicolumn{5}{|c|}{ Line parameters } & \multirow[t]{2}{*}{$\chi^{2} /$ d.o.f. } & \multirow[t]{2}{*}{ F-test } \\
\hline & & $E(\mathrm{keV})$ & $\sigma(\mathrm{keV})$ & $\theta(\mathrm{deg})$ & Flux & $E W(\mathrm{eV})$ & & \\
\hline PL & $1.56 \pm 0.04$ & - & - & - & - & - & $634.0 / 621$ & - \\
\hline PL + zgauss & $1.57 \pm 0.04$ & 6.4 (f) & 0.01 (f) & - & $2.2 \pm 1.2$ & $54 \pm 29$ & $624.5 / 620$ & $99.78 \%$ \\
\hline PL + zgauss & $1.57 \pm 0.04$ & $6.33 \pm 0.05$ & $<0.10$ & - & $2.9 \pm 1.4$ & $69_{-31}^{+36}$ & $619.6 / 618$ & $99.74 \%$ \\
\hline PL + DISKLINE $^{(a)}$ & $1.58 \pm 0.04$ & $6.40 \pm 0.05$ & - & $<22$ & $3.9 \pm 1.7$ & $107 \pm 46$ & $619.3 / 618$ & $99.77 \%$ \\
\hline $\mathrm{PL}+\mathrm{LAOR}^{(b)}$ & $1.58 \pm 0.04$ & $6.40_{-0.10}^{+0.05}$ & - & $<22$ & $4.4 \pm 2.1$ & $119_{-55}^{+59}$ & $621.7 / 618$ & $99.30 \%$ \\
\hline
\end{tabular}

the one found in general in radio-quiet quasars (Porquet et al. $2004 \mathrm{a} ;\left\langle\Gamma_{0.3-2 \mathrm{keV}}\right\rangle=2.43$ with a dispersion of 0.35$)$. The inferred $0.2-2 \mathrm{keV}$ unabsorbed flux is $2.9 \times 10^{-12} \mathrm{erg} \mathrm{cm}^{-2} \mathrm{~s}^{-1}$ $\left(L=1.1 \times 10^{44} \mathrm{erg} \mathrm{s}^{-1}\right)$. However, adding a narrow $(\sigma=10 \mathrm{eV})$ Gaussian line, we found a significant improvement to the fit, with $\chi^{2} /$ d.o.f. $=339.4 / 353$ (as a comparison, Monte Carlo simulations, as described in Sect. 3.2, give $>99.9 \%$ ). The energy of the line is $556 \pm 15 \mathrm{eV}$ and its equivalent width $(E W)$ is $15.9_{-5.6}^{+4.5} \mathrm{eV}$. These parameters are consistent with the position of the He-like O VII triplet between 561-574 eV. We also fit the data assuming two absorption edges, and we again found a significant improvement to the fit, with $\chi^{2} /$ d.o.f. $=339.9 / 351$ (F-test $>99.99 \%$ ). The energies (and optical depths, $\tau$ ) of the two edges are $E=0.714_{-0.032}^{+0.030} \mathrm{keV}(\tau=0.16 \pm 0.06)$ and $E=0.953_{-0.140}^{+0.055} \mathrm{keV}(\tau=0.10 \pm 0.05)$ respectively. The energy of the first edge is consistent with the rest-frame energy of the O VII edge $(0.740 \mathrm{keV})$. The second edge is not compatible with the rest-frame of the O VIII edge $(0.871 \mathrm{keV})$ or of Ne IX $(1.196 \mathrm{keV})$, but could be one of these ions with a velocity shift or perhaps a blend of the two edges. It also could be due the presence of absorption lines, for example, the Fe L complex seen in absorption in several AGN (e.g., Sako et al. 2001). Unfortunately the present RGS data do not have good enough $S / N$ to determine the physical nature of this feature. However, as we will discuss below, the whole average spectrum could be equally well explained either by X-ray ionized reflection on the accretion disk surface, by a warm absorber-emitter plus power-law model, or by a smeared absorption/emission from a high velocity outflow.

\subsubsection{The Fe $\mathrm{K} \alpha$ complex}

As shown in Fig. 1, there is the presence of a Fe $\mathrm{K} \alpha$ complex between 5.4 and $6.4 \mathrm{keV}$ in the quasar frame. Therefore, we fit the $2-12 \mathrm{keV}$ energy range with an absorbed power-law continuum and three possible profiles for the iron line; either (i) a simple Gaussian (ZGAUSS in XSPEC), (ii) a line profile corresponding to emission from a relativistic accretion disk around a non-rotating black hole (DISKLINE; Fabian et al. 1989), or (iii) emission from around a maximally rotating black hole (LAOR; Laor 1991). The spectral fits were improved by the addition of the iron line, when modeled either by a Gaussian line or by a relativistic accretion disk-line profile, with a significance greater than or equal to $99.3 \%$ from an F-test (see Table 2). The line, if modeled by a Gaussian, appears to be more likely narrow $(\sigma<0.11 \mathrm{keV})$ and produced by neutral to moderately ionized iron, with $E=6.34 \pm 0.05 \mathrm{keV}$. If the line is due to a relativistic accretion disk, the inclination of the disk is constrained to be less than about $20^{\circ}$. The line could be associated with X-ray fluorescence from the molecular torus. However, we will see below with time-resolved spectroscopy that the situation is more complex, with the possible presence of two non-simultaneous transient iron lines at $\sim 5.5 \mathrm{ks}$ and $\sim 6.3-6.4 \mathrm{keV}$ appearing during the first $5.5 \mathrm{ks}$ and last $16 \mathrm{ks}$ of the observation, respectively.

\subsubsection{The broad-band spectrum: $0.2-12 \mathrm{keV}$}

The $0.2-12 \mathrm{keV}$ energy range is well-fit by the combination of a blackbody and a power-law component. We find $N_{\mathrm{H}}^{\mathrm{int}}<$ $2.4_{-0.4}^{+0.5} \times 10^{20} \mathrm{~cm}^{-2}, k T_{\mathrm{bb}}=149_{-17}^{+20} \mathrm{eV}$ and $\Gamma=1.62_{-0.14}^{+0.28}$ $\left(\chi^{2} /\right.$ d.o.f. $\left.=1008.7 / 977\right)$. A broken power-law model also gave a good fit, with $N_{\mathrm{H}}^{\mathrm{int}}=2.9_{-1.1}^{+1.0} \times 10^{20} \mathrm{~cm}^{-2}, \Gamma_{\mathrm{soft}}=2.04_{-0.09}^{+0.08}, \Gamma_{\text {hard }}=$ $1.59_{-0.04}^{+0.03}$, and $E_{\text {break }}=1.64_{-0.13}^{+0.26} \mathrm{keV}\left(\chi^{2} /\right.$ d.o.f. $\left.=995.8 / 977\right)$.

In order to obtain a more physical representation of the soft excess, we have also tested multi-temperature disc models, which may be expected if the soft X-ray excess originates via thermal emission from the inner accretion disc in PG 1416-129. The DISKBB (non-relativistic) and DISKPN (relativistic) models were used, together with a power-law to model the hard X-ray emission above $2 \mathrm{keV}$. Equally good fits are obtained for both models. For the first model, we found $k T_{\text {diskbb }}=211_{-26}^{+33} \mathrm{eV}$ and $\Gamma=1.60 \pm 0.03\left(\chi^{2} /\right.$ d.o.f. $\left.=996.0 / 977\right)$, and similar parameters were found for the second model: $k T_{\text {diskpn }}=200_{-24}^{+32} \mathrm{eV}$ and $\Gamma=1.60 \pm 0.03\left(\chi^{2} /\right.$ d.o.f. $\left.=996.3 / 977\right)$. The inner disc temperatures obtained through either of these models are much larger than the temperature at $3 R_{\mathrm{S}}\left(R_{\mathrm{S}}=2 G M / c^{2}\right)$ expected from a standard steady state $\alpha$ thin accretion disc, i.e. $8-10 \mathrm{eV}$, (Shakura \& Sunyaev 1973), assuming a black hole mass of about $3-6 \times 10^{8} M_{\odot}$. This result is consistent with the temperature found in other low-redshift AGN, with a $100-200 \mathrm{eV}$ temperature component being too hot to be the direct thermal disk emission as inferred from the soft X-ray spectra (Gierliński \& Done 2004; Porquet et al. 2004a; Piconcelli et al. 2005).

The whole spectrum could be explained with a warm absorber/emitter combined with a power law continuum. Therefore, we used a grid of models generated by the XSTAR (version 211) photoionization code (Kallman et al. 1996) to fit the EPIC-pn data. A one zone model was adopted (covering the range of parameters $1<\log \xi<5$ and $21<\log N_{\mathrm{H}}^{\mathrm{warm}}<24$ ). Solar elemental abundances were assumed. A low turbulence velocity of $200 \mathrm{~km} \mathrm{~s}^{-1}$ was used, and the covering factor was fixed to unity. The warm absorber column density $\left(N_{\mathrm{H}}^{\mathrm{warm}}\right)$ was fixed to the lowest value in the XSTAR grid, $10^{21} \mathrm{~cm}^{-2}$, while the ionization parameter $(\xi)$ was left free. A good fit was obtained $\left(\chi^{2} /\right.$ d.o.f. $\left.=1077.6 / 975\right)$ with $N_{\mathrm{H}}^{\text {int }}<1.6 \times 10^{19} \mathrm{~cm}^{-2}$, $\Gamma=1.69 \pm 0.02, \log \xi=1.90_{-0.10}^{+0.03} \mathrm{erg} \mathrm{cm} \mathrm{s}^{-1}$. In this model, the photoionized emission from the warm absorber represents about $7 \%$ of the total flux in the $0.2-2 \mathrm{keV}$ energy range. 


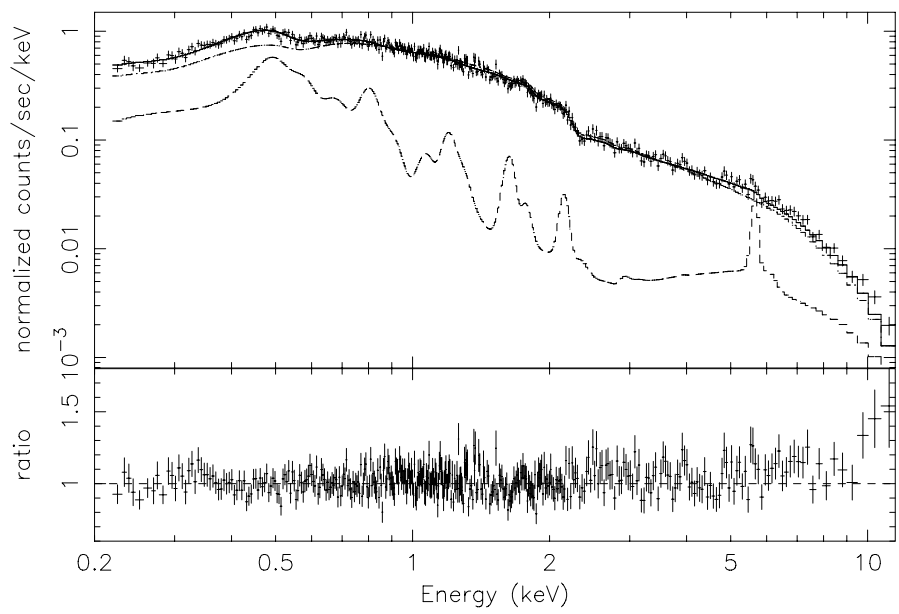

Fig. 2. The time-averaged pn spectrum of PG 1416-129 (in the observed frame). A relativistically blurred photo-ionized disc model (REFLION: Ross \& Fabian 2005) has been fit, and which provides a good representation of the whole energy band. A primary power-law component is taken into account in the model. Both component models are displayed without blurring in the upper panel. See text for the values of the fit parameters. For presentation only, the data have been re-binned into groups of 10 bins, after group of a minimum of 20 counts per bin is used for the fit.

We then tested the possibility that the whole spectrum could be explained by X-ray ionized reflection on the accretion disk surface (REFLION model in XSPEC; Ross \& Fabian 2005). Ross \& Fabian (2005) have shown that this model can explain the overall spectrum from $0.2-12 \mathrm{keV}$ in several AGN, without invoking ad-hoc components such as a blackbody (see also Crummy et al. 2006). One signature of ionized reflection is the soft excess emission which occurs in the $0.2-2 \mathrm{keV}$ band due to lines and bremsstrahlung emission from the hot surface layers. It creates a bump in the relativistically blurred spectrum which, when convolved with the XMM-Newton pn response matrix, is well fit by a blackbody of temperature $150 \mathrm{eV}$. The iron abundance $\left(A_{\mathrm{Fe}}\right)$ is from Morrison \& McCammon (1983) in this model. The REFLION model covers a large range of parameters: $30 \leq \xi \leq$ $10000 \mathrm{erg} \mathrm{cm} \mathrm{s}^{-1}$ (ionization parameter), $0.1 \leq A_{\mathrm{Fe}} \leq 10.0$ (relative iron abundance), and $1.0 \leq \Gamma \leq 3.0$. We also took into account the relativistic effect due to relativistic motion in the inner part of the accretion disc, by blurring the spectrum with a Laor line profile (KDBLUR; Ross \& Fabian 2005; Crummy et al. 2006). In the blurred reflection model, the physical lower limit of the inner radius $\left(R_{\text {in }}\right)$ was set at $1.235 R_{\mathrm{g}}$, i.e. corresponding to the innermost stable orbit around a maximally rotating black hole. We fixed the disc emissivity index to -3 . We obtained a very good fit $\left(\chi^{2} /\right.$ d.o.f. $\left.=980.9 / 974\right)$ to the data with the following parameters: $N_{\mathrm{H}}^{\text {int }}=3.6 \pm 1.3 \times 10^{20} \mathrm{~cm}^{-2}, R_{\mathrm{in}}=3.1_{-1.0}^{+1.6} R_{\mathrm{g}}$ $\left(R_{\mathrm{g}}=G M / c^{2}\right), \theta=21.1_{-18.8}^{+10.2 \circ}, A_{\mathrm{Fe}}=0.92_{-0.31}^{+0.45}, \Gamma=1.78 \pm 0.04$, and $\xi=38.0_{-5.1}^{+12.8} \mathrm{erg} \mathrm{cm}^{-1} \mathrm{~s}^{-1}$ (see Fig. 2). No additional absorption or emission features in the soft band were required. As shown in Fig. 2, the reflection emission is significant in the very soft part of the spectrum, especially near $0.4-0.5 \mathrm{keV}$ (observed frame), where a feature at $556 \mathrm{eV}$ has been detected using a simple power-law plus a narrow Gaussian (see Sect. 3.1.1). The fraction of the reflection emission to the total emission is only about $21 \%$ in the $0.2-12 \mathrm{keV}$ (similar values are found in the $0.2-2 \mathrm{keV}$ and $2-10 \mathrm{keV}$ energy ranges). The inner radius found here is compatible with a rotating black hole. The fit parameters found here are compatible to those determined in the large sample of type I AGN from Crummy et al. (2006). The powerlaw index is in the low range value of the sample but not as low than that found for Mrk $359(\Gamma=1.49 \pm 0.06)$, which is an extreme NLS1.

An alternative possibility is that the soft X-ray excess is an artifact of soft X-ray absorption and emission produced by a high velocity outflow, as shown by Gierliński \& Done (2004) and Schurch \& Done (2006), who model the X-ray spectra of other PG quasars observed by XMM-Newton. Therefore we fit the data with smeared ionized absorption and emission, using the same grid of models generated by the XSTAR photoionization code used for the warm absorber-emitter model used above. We fixed $N_{\mathrm{H}}^{\text {warm }}$ to $10^{21} \mathrm{~cm}^{-2}$. We obtained a very good fit to the data $\left(\chi^{2} /\right.$ d.o.f. $\left.=990.8 / 975\right)$, with a smearing velocity of $0.18 \pm 0.02 \mathrm{c}$, $N_{\mathrm{H}}^{\mathrm{int}}=9.0_{-3.9}^{+2.9} \times 10^{19} \mathrm{~cm}^{-2}, \log \xi=2.31_{-0.32}^{+0.15} \mathrm{erg} \mathrm{cm}^{-1} \mathrm{~s}^{-1}$, and $\Gamma=1.59_{-0.03}^{+0.02}$.

As we will see in the next section, an additional physical process, such as hot spots due to X-ray flares, is required to explain the two possible transient Fe K $\alpha$ lines observed (Sect. 3.2).

\subsection{XMM-Newton time-resolved spectroscopy}

To test possible rapid energy shifts of the iron line, we have created X-ray intensity maps in the energy-time plane using the pn data. Photons from the source cell were accumulated in pixels in the energy-time plane. The pixel distribution was smoothed in energy by the instrumental resolution, using a Gaussian of $140 \mathrm{eV}$ (appropriate for the single plus double events with the latest calibration), and smoothed in time using a top-hat function of width $10 \mathrm{ks}$. Each time-slice was background-corrected by subtracting a time-dependent background spectrum measured in an off-source region on the same detector chip as the source. The source continuum was modeled as an absorbed power-law, with variable amplitude and slope but time-invariant absorption column density. This continuum was subtracted, leaving positive and negative residuals that comprise noise plus any emission or absorption components on top of the continuum. More information about this method can be found in Turner et al. (2006). The "signal-to-noise" $(S / N)$ map presented in Fig. 3 is the ratio of the fluctuation amplitude to the calculated noise.

The highest $S / N$ observed is about 4.5 . We observe the presence of an excess with $S / N \geq 3.5$ at $\sim 5.5 \mathrm{keV}$ in the quasar frame at the beginning of this observation. Then this feature disappears. At the end of this observation, there is the presence of an excess near $6.3 \mathrm{keV}$ (in the quasar frame) and then at lower significance $(S / N \leq 3.5)$ a hint of a shift to $6.4 \mathrm{keV}$ of the centroid of the line. In order to characterize these features, which are most probably associated with $\mathrm{Fe} \mathrm{K} \alpha$ line emission, we have split the observation into three sub-spectra (the corresponding time ranges are illustrated on Fig. 3):

- 1 This corresponds to the presence of the $5.5 \mathrm{keV}$ feature with $S / N \geq 3.5$ (from $t=0 \mathrm{ks}$ to $t=5.5 \mathrm{ks}$ );

-2 This corresponds to the absence of any significant long-lived excess feature (from $t=5.5 \mathrm{ks}$ to $32 \mathrm{ks}$ );

- 3 This corresponds to the appearance and presence of the feature near $6.3-6.4 \mathrm{keV}$.

First, we rebinned the sub-spectra, grouping every $80 \mathrm{eV}$ (16 channels). This was chosen to sample the resolution of the pn detector (i.e. about 2 spectral bins per $F W H M$ resolution element). We then fit them with a power-law continuum over the $2-10 \mathrm{keV}$ energy range. The parameter fits are reported in Table 3 and the spectral fits are displayed in Fig. 4. For 


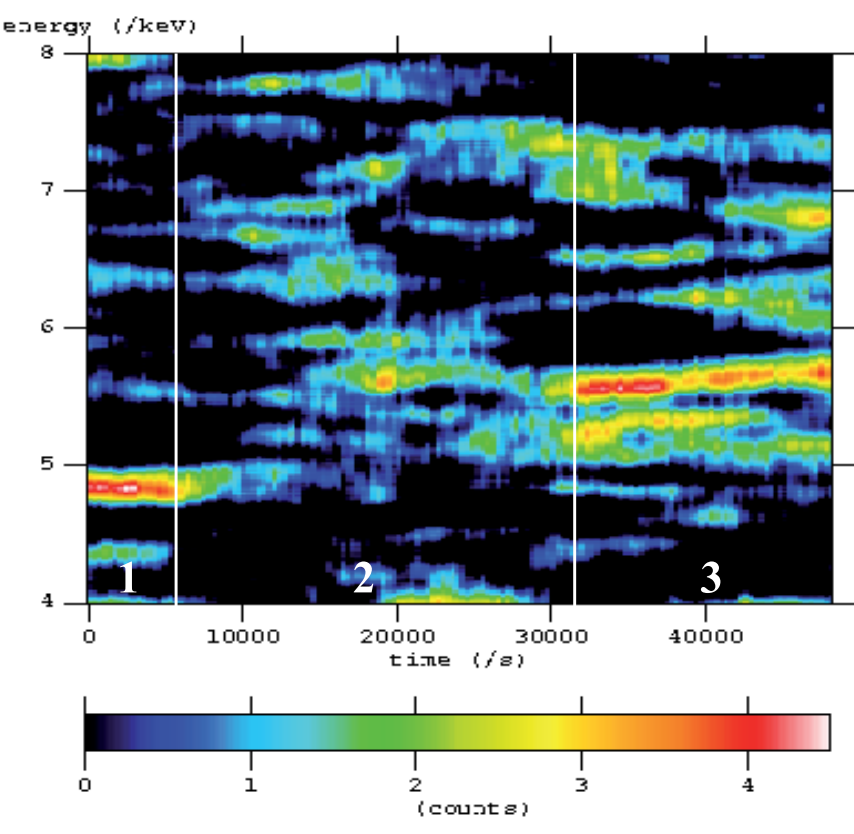

Fig. 3. The signal-to-noise deviations (in $\sigma$ ) of the $\mathrm{Fe} \mathrm{K} \alpha$ line above the power-law continuum in the energy-time plane. The energy axis is given in the observed frame. The colour-scale represents excess signal/noise in the line counts above the fitted continuum. Energy and time are oversampled by a factor of 10 . Data are top-hat smoothed by $10 \mathrm{ks}$ in time and Gaussian smoothed with $F W H M=0.14 \mathrm{keV}$ in energy. The regions 1-3 correspond to the time selections used for the time-resolved spectroscopic analysis. See text for explanation.

Table 3. Best-fit spectral parameters for the three pn sub-spectra in the $2-10 \mathrm{keV}$ energy range. Column (1): number of the corresponding sub-spectrum (the extraction time range is reported in Fig. 3). Column (2): model used. PL: power-law; zgauss: Gaussian line ( $\sigma$ fixed to $10 \mathrm{eV})$; AN: narrow diskline annulus $\left(\Delta R=1 R_{\mathrm{g}}\right.$; other diskline parameters are discussed in the text, see Sect. 3.2). Column (3): power-law index. Column (4): unabsorbed $2-10 \mathrm{keV}$ flux $\left(\times 10^{-12} \mathrm{erg} \mathrm{cm}^{-2} \mathrm{~s}^{-1}\right)$. Column (5): line parameters.

\begin{tabular}{llcccc}
\hline \hline No. & Model & $\Gamma$ & $F_{2-10}^{\text {unabs }}$ & $\begin{array}{c}E_{\text {line }}(\mathrm{keV}) \\
E W(\mathrm{eV})\end{array}$ & $\chi^{2} /$ d.o.f. \\
\hline 1 & PL & $1.61 \pm 0.11$ & $3.2 \pm 0.5$ & - & $83.8 / 97$ \\
& PL+zgauss & $1.64 \pm 0.11$ & - & $\begin{array}{l}5.47 \pm 0.04 \\
194 \pm 89\end{array}$ & $71.0 / 95$ \\
& & & $194 \pm 0$. & $98.8 / 97$ \\
\hline 2 & PL & $1.51 \pm 0.05$ & $3.3 \pm 0.2$ & - & $117.9 / 97$ \\
\hline 3 & PL & $1.61 \pm 0.06$ & $3.1 \pm 0.2$ & - & \\
& PL+zgauss & $1.62 \pm 0.06$ & - & $6.35 \pm 0.05$ & $102.5 / 95$ \\
& & & & $128 \pm 53$ & \\
& PL+AN & $1.64 \pm 0.06$ & - & $6.40(\mathrm{f})$ & $95.0 / 93$ \\
& & & & $287 \pm 100$ & \\
\hline
\end{tabular}

sub-spectra 1 and 3, an emission feature is observed. For subspectrum 1, the addition of a narrow Gaussian line (width fixed to $10 \mathrm{eV}$ ) with a centroid energy of $\sim 5.5 \mathrm{keV}$ improved the fit. Allowing the line width to vary, we found an upper limit of $94 \mathrm{eV}$. For sub-spectrum 3, the addition of a narrow Gaussian (width fixed to $10 \mathrm{eV}$ ) with a centroid energy of about $6.35 \mathrm{keV}$ also improved the fit. If we allow the line width to be a free parameter, the upper limit is rather large $(\sigma \leq 0.4 \mathrm{keV})$ due to the presence of the red wing-like feature seen below $6.4 \mathrm{keV}$ (see Fig. 4). A narrow disc annulus at $6.4 \mathrm{keV}\left(\Delta R=1 R_{\mathrm{g}}\right.$, emissivity index fixed to -2$)$ provides a superior fit. The best fit parameters are $\Gamma=1.64 \pm 0.06, R_{\mathrm{in}}=34_{-8}^{+5} R_{\mathrm{g}}, \theta=17 \pm 3^{\circ}$ and

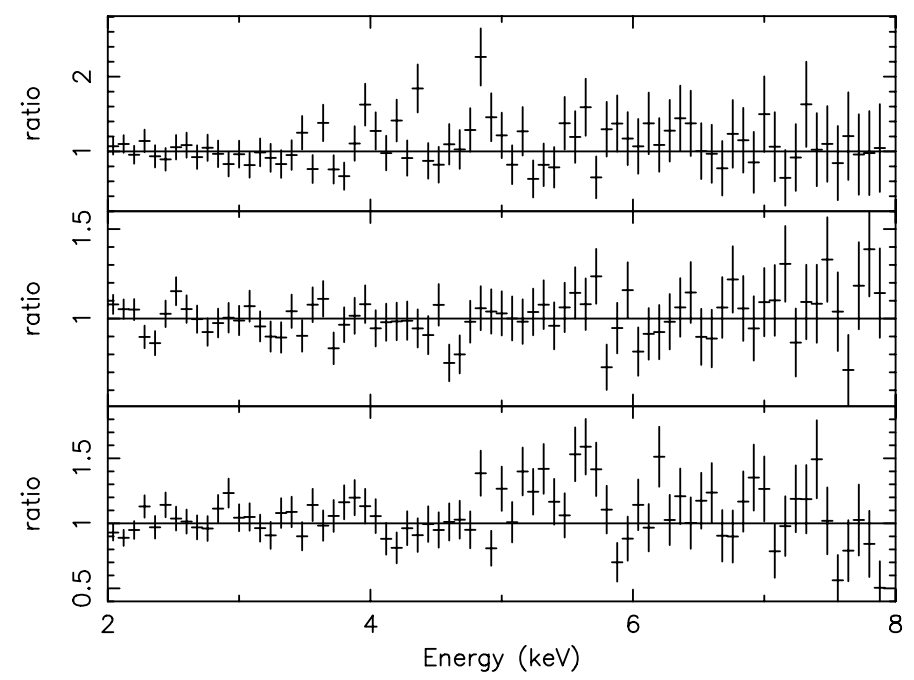

Fig. 4. The three pn time-resolved spectroscopy spectra (in the observed frame) obtained by splitting the average spectra according to the time regions shown in Fig. 3. The three sub-spectra have been fit with a power-law continuum model. Here, $5.67 \mathrm{keV}$ in the observed frame corresponds to $6.4 \mathrm{keV}$ in the rest frame. Notice the residuals present at $4.8 \mathrm{keV}$ and $5.6 \mathrm{keV}$ in segments 1 (upper panel) and 3 (lower panel), respectively.

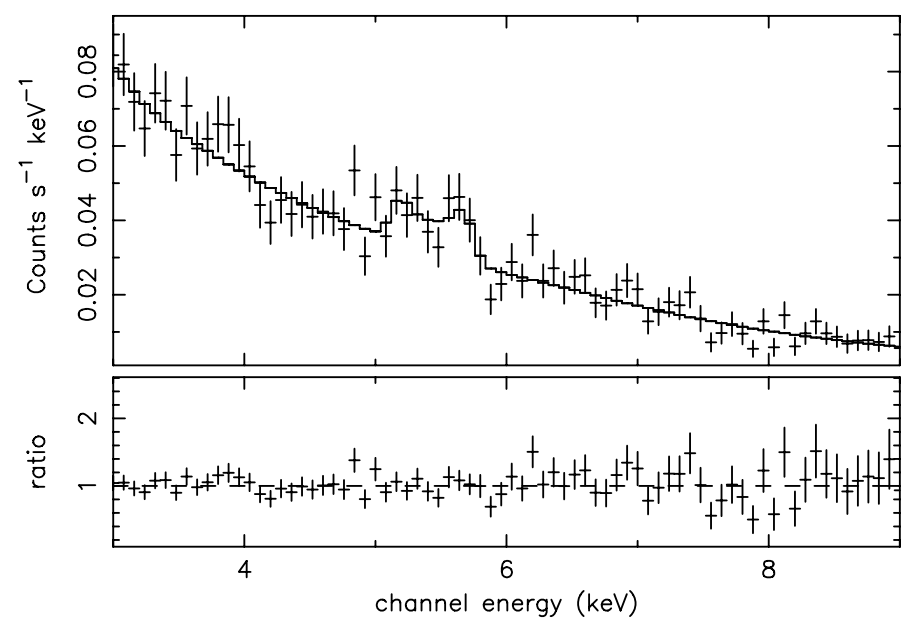

Fig. 5. The pn sub-spectrum 3 of PG 1416-129 (in the observed frame). A power law continuum plus a DISKLINE model have been fit. A narrow annulus is assumed with $\Delta R=1 R_{\mathrm{g}}$. See text for the best fit parameter values.

$E W=287 \pm 100 \mathrm{eV}$ (Fig. 5). Letting the value of the accretion disc outer radius free to vary, we find an upper limit of $84 R_{\mathrm{g}}$, corresponding to a $\Delta R$ of the annulus value between 1 and $58 R_{\mathrm{g}}$ (taking into account the lower limit on $R_{\text {in }}$ ).

As discussed by Protassov et al. (2002) (see also Porquet et al. 2004b), the F-test can overestimate the true detection significance of a line if there was no previous expectation of the line. One must account for the number of resolution elements $N$ over the bandpass of interest. The probability of detecting a feature at any energy in this range is obtained using $\left(P_{1}\right)^{N}$, where $P_{1}$ is the probability of finding a feature at an expected line energy (equal to one minus the null hypothesis probability). Assuming 21 resolution elements between $4-7 \mathrm{keV}$, the significance probabilities for the 5.5 and $6.3 \mathrm{keV}$ lines become $98.1 \%$ and $99.2 \%$, respectively. We also carried out a more rigorous test of the significance of the lines using Monte Carlo simulations to assess 
the likelihood that the lines could be an artefact of photon noise (e.g., Porquet et al. 2004b). For our null hypothesis, we assumed that the spectrum is simply an absorbed power-law continuum, with the same parameters as the absorbed power-law model fit to the real data. We used the XSPEC FAKEIT NONE command to create a fake spectrum, and then, to account for the uncertainty in the null hypothesis model itself, we refit the null hypothesis model and ran FAKEIT NONE a second time on the "refit" null hypothesis model. Both FAKEIT NONE commands were run with the photon statistics appropriate for a $5.5 \mathrm{ks}$ exposure (segment 1) or $16 \mathrm{ks}$ exposure (segment 3). We grouped each faked spectrum, rebinning every $80 \mathrm{eV}$. We then added a narrow $(\sigma=10 \mathrm{eV})$ Gaussian to the fit for the $5.5 \mathrm{keV}$ line, or a diskline annulus, with the values of the inner and outer radii, emissivity index, and inclination angle fixed to that of the observed spectrum, for the $6.3 \mathrm{keV}$ line. We searched over 4.0 to $7.0 \mathrm{keV}$ (observed frame) in increments of $0.1 \mathrm{keV}$, fitting separately each time to ensure that the lowest value of $\chi^{2}$ was found. We compared the minimum value of $\chi^{2}$ obtained with the corresponding $\chi^{2}$ of the null hypothesis fit. This process was repeated 1000 times, yielding a cumulative frequency distribution of the simulated $\Delta \chi^{2}$ expected for a blind line search. The inferred probabilities to reject the null hypothesis are $98.8 \%$ and $99.7 \%$ for the $5.5 \mathrm{keV}$ and $6.3 \mathrm{keV}$ lines, respectively. However, since we have divided the whole observation into three sub-spectra, the null hypothesis probabilities have to be multiplied by a factor of three, leading to $96.4 \%$ and $99.1 \%$ for the detection confidence level of these transient lines. While the detection of the $5.4 \mathrm{keV}$ feature is statistically marginal, it should be noted that the $6.3 \mathrm{keV}$ feature is observed at an energy where a iron $\mathrm{K} \alpha$ diskline would be expected a priori.

The three sub-spectra display no significant flux variations: $F_{2-10}^{\text {unabs }}$ is consistent with being constant. As far as the continuum photon index is concerned, sub-spectra 1 and 3 have consistent values, but sub-spectrum 2 is slightly flatter; the photon index when the diskline annulus model is applied to sub-spectrum 3 is not consistent at $90 \%$ confidence with that obtained in subspectrum 2, where no emission line was found. However, we can use Monte Carlo simulations to quantify the expected spread in the measured photon index, given an assumed intrinsic constant photon index. Specifically, the null hypothesis model in the Monte Carlo procedure above assumed a single value for the intrinsic photon index; using those same simulated spectra, we find a standard deviation in the measured photon index of 0.165 (due to the moderate signal/noise). This is higher than the maximum $\Delta \Gamma$ of 0.13 between the best-fit photon indices for sub-spectra 3 and 2 . Therefore, there is no evidence for the appearance of the line (or lack thereof) to be correlated with values of the photon index.

\section{Summary and discussion}

In this section, we summarize the main results of our analysis of the July 2004 XMM-Newton/EPIC observation of PG 1416-129. - A X-ray flat RQQ object.

With the present 2004 XMM-Newton observation, we found that its $2-5 \mathrm{keV}$ power-law index $(\Gamma \sim 1.6)$ is similar to values found for radio-loud objects $(\Gamma=1.74$ with a standard deviation of 0.03; Porquet et al. 2004a), but by far much harder than the value commonly seen in radio-quiet quasars: $\Gamma=1.90$ (with a standard deviation of 0.27 ) and $\Gamma=2.37$ (with a standard deviation of 0.11) for Broad Line quasars and Narrow Line quasars, respectively (Porquet et al. 2004a). This result confirms the behavior seen in previous observations, with the exception of the 1994 ASCA observation, as reported in Table 1.

- Is PG1416-129 a NLS1?

PG 1416-129 could be associated with the NLS1 class of objects, given the value of $F W H M(\mathrm{H} \beta)$ observed in 2000 by Sulentic et al. (2000).

NLS1s are defined according to the following characteristics:

- FWHM(H $\beta)$ less than $2000 \mathrm{~km} \mathrm{~s}^{-1}$; strong optical Fe II multiplets; [O III] $\lambda 5007 \AA / \mathrm{H} \beta_{\mathrm{n}} \leq 3$ (Osterbrock \& Pogge 1985; Goodrich 1989). The 2000 optical spectrum of PG1416-129, however, showed only a weak Fe II multiplet, with [O III] $\lambda 5007 \AA / \mathrm{H} \beta \sim 13.9$ (Sulentic et al. 2000).

- steep, soft X-ray excess (Puchnarewicz et al. 1992; Boller et al. 1996); rapid soft/hard X-ray variability (Leighly 1999). During the 2004 XMM-Newton observation, however, neither significant X-ray variability nor a steep soft excess were observed.

- less massive black holes with higher Eddington ratios, suggesting that they might be in the early stage of AGN evolution (Grupe 1996; Mathur 2000; Bian \& Zhao 2003). As reported in the introduction, however, this object has a black hole mass of about $3-6 \times 10^{8} M_{\odot}$, which is higher than the mass found in typical NLS1s. Furthermore, its accretion rate with respect to Eddington is only 0.12 .

Therefore it appears that PG 1416-129 is not a genuine NLS1 and instead the narrow $\mathrm{H} \beta$ width is caused by the variations in the broad component of this line.

- The soft X-ray spectrum.

In addition to its flat $2-10 \mathrm{keV}$ photon index, this object shows a much weaker soft excess than generally observed for BroadLine quasars and Narrow-Line quasars. Some additional features seem to be present in the $0.2-2 \mathrm{keV}$ energy range. For example, the addition of a Gaussian emission line at $556 \pm 15 \mathrm{eV}$ improved the fit very significantly, with an F-test significance of $>99.99 \%$. However, we have obtained a fit with the same statistical quality by adding two absorption edges. Unfortunately the present RGS data do not have sufficient signal to noise ratio in order to determine the physical nature of this feature, if real. The whole average spectrum is very well fit by a model with X-ray ionized reflection on the accretion disk surface, with no additional soft $\mathrm{X}$-ray absorption and/or emission features required. However other models, such as absorption and/or re-emission from a high velocity outflow (or simple photoionized emission from distant matter) could also explain the weak soft excess below $1 \mathrm{keV}$, as discussed in detail for other PG quasars by Gierliński \& Done (2004).

- Discovery of two possible transient $\mathrm{Fe} \mathrm{K} \alpha$ lines

In the time-averaged spectrum, a Fe $\mathrm{K} \alpha$ complex is seen between 5.5 and $6.4 \mathrm{keV}$, with significant residuals at about $6.4 \mathrm{keV}$. If the line is due to a relativistic accretion disk, the inclination of the disk is constrained to be less than about $20^{\circ}$. If the line is variable, this would rule out X-ray fluorescence from distant matter such as the molecular torus. Indeed, time-resolved spectroscopy has revealed that the emission complex is more likely due to the presence of two transient iron lines. The first iron line has a redshifted energy centered at $\sim 5.5 \mathrm{keV}$ and was present during the first $\sim 5.5 \mathrm{ks}(E W \sim 167 \mathrm{eV})$ of this observation with a 96.4\% confidence level according to Monte-Carlo simulations (see Sect. 3.2); after $5.5 \mathrm{ksec}$, it disappears sharply. The second, more significant transient iron line appears about $32 \mathrm{ks}$ after the beginning of this observation with a centroid energy at $\sim 6.3 \mathrm{keV}$ $(E W \sim 250 \mathrm{eV})$ and a duration of at least $\sim 16 \mathrm{ks}$ with a $99.1 \%$ 
confidence level according to Monte-Carlo simulations. There is a hint of a slight shift in its centroid energy to $\sim 6.4 \mathrm{keV}$ during the last $10 \mathrm{ks}$ of the observation. Other narrow, highly-redshifted iron lines $(\sim 4.5-6 \mathrm{keV})$ in AGN have already been reported: e.g., NGC 3516 (Turner et al. 2002), ESO 113-G010 (Porquet et al. 2004b), Mrk 766 (Turner et al. 2004), AX J0447-0627 (Della Ceca et al. 2005), and PG 1425+267 (Miniutti \& Fabian 2006). Localized spots or narrow annuli which occur on the surface of an accretion disk following its illumination by flares have been proposed to explain these features (e.g., Nayakshin 2000; Turner et al. 2002; Dovčiak et al. 2004; Pecháček et al. 2005). Such lines may arise in the accretion disk, sporadically illuminated by "hotspots", as regions of magnetic reconnection illuminate very small areas of the accretion flow. Presumably the hotspot co-rotates with the disk. While the small line width implies that the emitting region must be small and detected only during a fraction of the whole orbit, large spots, meanwhile, would produce broader features and they would be prone to rapid destruction. Here the features appear to be narrow, however their widths are not strongly constrained. Indeed, an annulus of up to $58 R_{\mathrm{g}}$ in size is allowed in segment 3 of the observation. If the transient lines are due to hotspots co-rotating with the disk, a lower limit of $16 \mathrm{ks}$ on the lifetime of the transient $6.3 \mathrm{keV}$ iron line feature would correspond to at least $80 \%$ and $2 \%$ of the whole orbit at $R=1.235 R_{\mathrm{g}}$ and $R=20 R_{\mathrm{g}}$, respectively (assuming a black hole mass of $3 \times 10^{8} M_{\odot}$ ). The large $E W$ of both transient features are consistent with the X-ray emission being located in a relatively compact region, e.g., from $\mathrm{X}$-ray flares. More complex geometries of the X-ray emitting region can be considered, e.g., spiral waves propagating across the accretion disc (e.g., Karas et al. 2001; Fukumura \& Tsuruta 2004), and density inhomogeneities, which can indicate turbulence driven by magnetorotational instability (Balbus \& Hawley 1991), or photon bubble instabilities (Arons 1992). As demonstrated by Ballantyne et al. $(2004,2005)$, photon bubble instabilities (which result on time-varying density homogeneities on scales smaller than the disk thickness) can explain rapid variability of emission lines, with $E W$ variations as large as $\sim 100 \mathrm{eV}$ that are independent of the illuminating continuum, as observed during the present observation. In addition, scenarios involving non-flat accretion disks, such as warped/corrugated accretion disks (e.g., Fabian et al. 2002; Miller \& Homan 2005), can be invoked to explain such features. However, as pointed out by Fabian et al. (2002), even a small corrugation of the accretion disc may result in $\Gamma>2$ and a strong reflection component in the observed spectrum, as observed in several NLS1 objects (e.g., 1H 0707-495). In the case of the present observation of PG 1416-129 such a scenario appears unlikely since neither a steep spectrum $(\Gamma \sim 1.6)$ nor a strong reflection component (the fraction of the $0.2-12 \mathrm{keV}$ reflection emission is only about $21 \%$ of the total emission, see Sect. 3.1.3) are observed.

Acknowledgements. The XMM-Newton project is an ESA Science Mission with instruments and contributions directly funded by ESA Member States and the USA (NASA). The XMM-Newton project is supported by the Bundesministerium für Wirtschaft und Technologie/Deutsches Zentrum für Luft- und Raumfahrt (BMWI/DLR, FKZ 50 OX 0001), the Max-Planck Society and the HeidenhainStiftung. We thank the anonymous referee for fruitful comments and suggestions. D.P. acknowledges grant support from an MPE fellowship.

\section{Appendix A: RXTE data}

PG 1416-129 was observed by RXTE on 1998 Aug. 21 from 06:25-10:42 UT, 1998 Aug. 22 from 06:54-08:06 UT, and
1998 Aug. 23 from 01:37-07:32 UT, for a total of $41.1 \mathrm{ks}$. Data were taken using RXTE's proportional counter array (PCA; Swank 1998), which consists of five identical collimated proportional counter units (PCUs). Data were collected from PCUs 0,1 , and 2, and reduced using standard extraction methods and FTOOLS v5.3.1 software. Data were rejected if they were gathered less than $10^{\circ}$ from the Earth's limb, if they were obtained within $30 \mathrm{~min}$ after the satellite's passage through the South Atlantic Anomaly, if ELECTRON0 > 0.1, or if the satellite's pointing offset was greater than 0.02 .

As the PCA has no simultaneous background monitoring capability, background data were estimated by using PCABACKEST V3.0 to generate model files based on the particle-induced background, SAA activity, and the diffuse X-ray background. The "L7-240" background models appropriate for faint sources were used. This background subtraction is the dominant source of systematic error in RXTE AGN monitoring data (e.g., Edelson $\&$ Nandra 1999). Counts were extracted only from the topmost PCU layer to maximize the signal-to-noise ratio. This reduction yielded a total of $22.0 \mathrm{ks}$ of good exposure time.

Response matrices were generated using PCARSP v.8.0. For the purposes of spectral analysis, data below $3.0 \mathrm{keV}$ were discarded in order to disregard PCA calibration uncertainties below this energy. The source was not well detected by the PCA above $\sim 14 \mathrm{keV}$; photons above this energy were discarded.

Fitting these RXTE data with an absorbed $\left(N_{\mathrm{H}}^{\mathrm{Gal}}\right)$ powerlaw continuum, we found a good fit with $\Gamma=1.17 \pm 0.15$ $\left(\chi^{2} /\right.$ d.o.f. $\left.=7.6 / 25\right)$. The inferred $2-10 \mathrm{keV}$ luminosity is $1.0 \times$ $10^{44} \mathrm{erg} \mathrm{s}^{-1}$.

\section{References}

Anders, E., \& Grevesse, N. 1989, Geochim. Cosmochim. Acta, 53, 197 Arnaud, K. A. 1996, in Astronomical Data Analysis Software and Systems V, ed. G. H. Jacoby, \& J. Barnes, ASP Conf. Ser., 101, 17

Arons, J. 1992, ApJ, 388, 561

Balbus, S. A., \& Hawley, J. F. 1991, ApJ, 376, 214

Ballantyne, D. R., Turner, N. J., \& Blaes, O. M. 2004, ApJ, 603, 436

Ballantyne, D. R., Turner, N. J., \& Young, A. J. 2005, ApJ, 619, 1028

Barkhouse, W. A., \& Hall, P. B. 2001, AJ, 121, 2843

Bian, W.-H., \& Zhao, Y.-H. 2003, PASJ, 55, 143

Boller, T., Brandt, W. N., \& Fink, H. 1996, A\&A, 305, 53

Boroson, T. A., \& Green, R. F. 1992, ApJS, 80, 109

Crummy, J., Fabian, A. C., Gallo, L., \& Ross, R. R.. 2006, MNRAS, 365, 1067 de Kool, M., \& Meurs, E. J. A. 1994, A\&A, 281, L65

Della Ceca, R., Ballo, L., Braito, V., \& Maccacaro, T. 2005, ApJ, 627, 706

Dickey, J. M., \& Lockman, F. J. 1990, ARA\&A, 28, 215

Dovčiak, M., Bianchi, S., Guainazzi, M., Karas, V., \& Matt, G. 2004, MNRAS, 350,745

Edelson, R., \& Nandra, K. 1999, ApJ, 514, 682

Fabian, A. C., Ballantyne, D. R., Merloni, A., et al. 2002, MNRAS, 331, L35

Fabian, A. C., Rees, M. J., Stella, L., \& White, N. E. 1989, MNRAS, 238, 729

Fukumura, K., \& Tsuruta, S. 2004, ApJ, 613, 700

George, I. M., Turner, T. J., Yaqoob, T., et al. 2000, ApJ, 531, 52

Gierliński, M., \& Done, C. 2004, MNRAS, 349, L7

Goodrich, R. W. 1989, ApJ, 342, 224

Grupe, D. 1996, Ph.D. Thesis

Hao, C. N., Xia, X. Y., Mao, S., Wu, H., \& Deng, Z. G. 2005, ApJ, 625, 78

Kallman, T. R., Liedahl, D., Osterheld, A., Goldstein, W., \& Kahn, S. 1996, ApJ, 465,994

Karas, V., Martocchia, A., \& Subr, L. 2001, PASJ, 53, 189

Laor, A. 1991, ApJ, 376, 90

Lawson, A. J., \& Turner, M. J. L. 1997, MNRAS, 288, 920

Leighly, K. M. 1999, ApJS, 125, 297

Mathur, S. 2000, MNRAS, 314, L17

Miller, J. M., \& Homan, J. 2005, ApJ, 618, L107

Miniutti, G., \& Fabian, A. C. 2006, MNRAS, 366, 115

Morrison, R., \& McCammon, D. 1983, ApJ, 270, 119

Nayakshin, S. 2000, ApJ, 540, L37

Osterbrock, D. E., \& Pogge, R. W. 1985, ApJ, 297, 166 
Pecháček, T., Dovčiak, M., Karas, V., \& Matt, G. 2005, A\&A, 441, 855

Piconcelli, E., Jimenez-Bailón, E., Guainazzi, M., et al. 2005, A\&A, 432, 15

Porquet, D., Reeves, J. N., O’Brien, P., \& Brinkmann, W. 2004a, A\&A, 422, 85

Porquet, D., Reeves, J. N., Uttley, P., \& Turner, T. J. 2004b, A\&A, 427, 101

Protassov, R., van Dyk, D. A., Connors, A., Kashyap, V. L., \& Siemiginowska, A. 2002, ApJ, 571, 545

Puchnarewicz, E. M., Mason, K. O., Cordova, F. A., et al. 1992, MNRAS, 256, 589

Reeves, J. N., \& Turner, M. J. L. 2000, MNRAS, 316, 234

Ross, R. R., \& Fabian, A. C. 2005, MNRAS, 358, 211

Sako, M., Kahn, S. M., Behar, E., et al. 2001, A\&A, 365, L168

Schurch, N. J., \& Done, C. 2006, ArXiv Astrophysics e-prints

Shakura, N. I., \& Sunyaev, R. A. 1973, A\&A, 24, 337
Staubert, R., \& Maisack, M. 1996, A\&A, 305, L41

Strüder, L., Briel, U., Dennerl, K., et al. 2001, A\&A, 365, L18

Sulentic, J. W., Marziani, P., Zwitter, T., Dultzin-Hacyan, D., \& Calvani, M. 2000, ApJ, 545, L15

Swank, J. H. 1998, Nucl. Phys. B Proc. Suppl., 69, 12

Turner, M. J. L., Abbey, A., Arnaud, M., et al. 2001, A\&A, 365, L27

Turner, T. J., Kraemer, S. B., \& Reeves, J. N. 2004, ApJ, 603, 62

Turner, T. J., Miller, L., George, I. M., \& Reeves, J. N. 2006, A\&A, 445, 59

Turner, T. J., Mushotzky, R. F., Yaqoob, T., et al. 2002, ApJ, 574, L123

Vestergaard, M. 2002, ApJ, 571, 733

Williams, O. R., Turner, M. J. L., Stewart, G. C., et al. 1992, ApJ, 389, 157

Wilms, J., Allen, A., \& McCray, R. 2000, ApJ, 542, 914 